\title{
High yields of aromatic monomers from acidolytic oxidation of Kraft lignin in a biphasic system: Supplementary Infor- mation (SI)
}

Katarzyna Arturi ${ }^{1, \star,}$, Thomas Rohrbach ${ }^{2}$, Frédéric Vogel $^{1,3}$, Saša Bjelic ${ }^{1, *}$

${ }^{1}$ Paul Scherrer Institute, Energy and Environment Division, Laboratory for Bioenergy and Catalysis, Forschungsstrasse 111, 5232 Villigen PSI, Switzerland

§Current address: Swiss Federal Institute of Aquatic Science and Technology (Eawag), Ueberlandstrasse 133, 8600 Dübendorf, Switzerland

${ }^{2}$ Paul Scherrer Institute, Energy and Environment Division, Laboratory for Catalysis and Sustainable Chemistry, Forschungsstrasse 111, 5232 Villigen PSI, Switzerland

${ }^{3}$ University of Applied Sciences Northwestern Switzerland (FHNW), Institute of Bioenergy and Resource Efficiency, Klosterzelgstrasse 2, 5210 Windisch, Switzerland ${ }^{\star}$ Corresponding author: kasia.arturi@eawag.ch, sasa.bjelic@psi.ch

\section{SI Materials}

Lignin used in this study was Kraft pine lignin Indulin ${ }^{\circledR}$ AT (Ingevity, 5255 Virginia Ave, North Charleston, SC, US) in the form of free-flowing brown powder with no insolubles in a warm aqueous solution of $5 \% \mathrm{NaOH}$ and the following characteristics: moisture 3.5 wt. $\%$, ash 1.9 wt. $\%$, average molar mass $8000 \mathrm{~g} / \mathrm{mol}$ as declared by the supplier. Solvents purchased from Sigma Aldrich included acetonitrile $(\mathrm{ACN})$, methanol $(\mathrm{MeOH})$, formic acid $(\mathrm{HCOOH})$, and high purity water. All were of high-performance liquid chromatography (HPLC) grade (purity $\geq 99.99 \%$ ). 1-octanol (99.5\%) was purchased from TCI and purified by three consecutive extractions in equal volumes of $0.05 \mathrm{HCl}$, followed by drying with an inert drying agent (sodium sulfate, $\mathrm{Na}_{2} \mathrm{SO}_{4}$ ) for 3 hours. Catalysts tested 
included: $\mathrm{FeCl}_{3}$ (iron(III) chloride from Sigma Aldrich, MW=162.20, CAS: 7705-08-0 ,

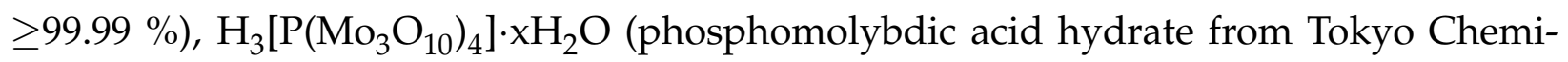
cal Industry, MW=1825.25 (anhydrous basis) g/mol, CAS: 51429-74-4, $\geq 99.99 \%$, designated as POM), and anthraquinone $\left(\mathrm{C}_{14} \mathrm{H}_{8} \mathrm{O}_{2}, \mathrm{MW}=208.21 \mathrm{~g} / \mathrm{mol}\right.$, CAS 84-65-1, designated $\mathrm{AQ}$ ). Concentrated $\mathrm{HCl}$ from Sigma Aldrich was used for adjustment of $\mathrm{pH}$ (37 wt.\%, hydrochloric acid, ACS reagent from Sigma Aldrich, MW 36.46, CAS: 7647-01-0). $\mathrm{O}_{2}(\geq 99.998 \%)$ were used for saturating the reaction vials prior to the experiments involving oxygen. $\mathrm{H}_{2} \mathrm{O}_{2}$ was used as a liquid oxidant (30 wt.\% in $\mathrm{H}_{2} \mathrm{O}$, ACS reagent from Sigma Aldrich, MW=34.04 g/mol, CAS: 7722-84-1). Unless stated otherwise, all commercially available chemicals were used without further purification.

\section{SI Experimental}

The microwave utilized a "very high absorbance" setting ( $90 \mathrm{~W}$ maximum power) and the following parameters: magnetic stirring $1200 \mathrm{rpm}, \mathrm{T}=170^{\circ} \mathrm{C}$, pre-stirring $15 \mathrm{~s}$, pressure 10 bar (generated autogenously). After the heating time of $2.5 \mathrm{~min}$ and holding time of 10-40 $\mathrm{min}$, the reaction mixture was cooled down rapidly with air $(1.5 \mathrm{~min})$ to room temperature. Figure S1 shows a typical temperature/pressure profile for the system. Reaction times under microwave conditions reflected holding times at the maximum set temperature and not the total irradiation times. The temperature was measured with an IR sensor on the outside of the reaction vessel. The gas products were not collected, as none were expected. In the runs with $\mathrm{O}_{2}$ as the oxidant, the sealed mixtures were saturated with oxygen for $15 \mathrm{~min}$ at $30 \mathrm{ml} / \mathrm{min}$ (oxygen concentrations were measured with OX-NP809408 micro-sensor with a needle for piercing the septum of a sealed Biotage vial from Unisense: $\mathrm{O}_{2, \mathrm{H}_{2} \mathrm{O}}=30 \mathrm{mg} / \mathrm{L}, \mathrm{O}_{2}, \mathrm{oct}=1.25 \mathrm{~g} / \mathrm{L}$ ). In the runs with Ar, the samples were purged with argon for $15 \mathrm{~min}$. Table 1 shows the full factor experimental design with combinations of parameters. The experiments were randomized to ensure the statistical independence of the obtained results. The reproducibility of the experimental procedure, 
assessed based on triplicates of the runs with oxygen, was below $10 \%$ (relative standard deviation). Blind runs without lignin were run to assess the stability of octanol at conversion conditions and as a baseline for the analytical procedure.

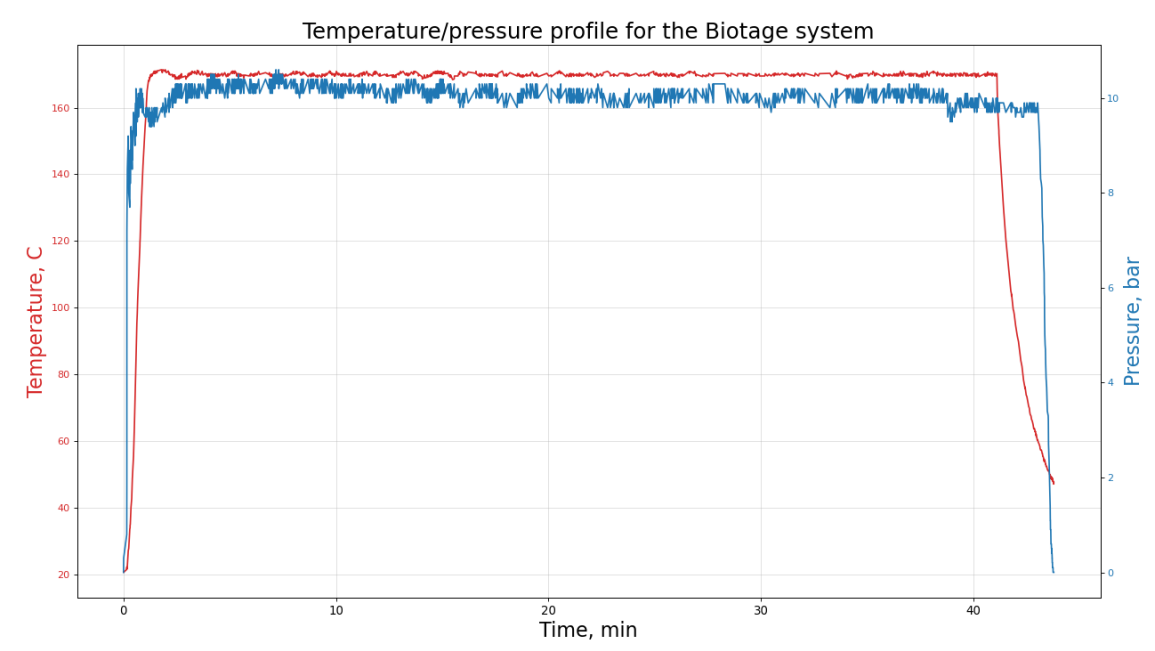

Figure S1: Temperature/pressure profiles for the Biotage microwave system.

Experimental conditions such as biphasic system composition, temperature, $\mathrm{pH}$, and reaction time were pre-selected based on previous work ${ }^{21}$ to promote acidolysis and oxidation while minimizing condensation. Octanol was selected based on its excellent solvent properties combining high solubility of aromatic oxygenates with low solubility for catalysts and lignin. Octanol is an inert green solvent (low volatility and toxicity, can be produced from biomass) that forms biphasic mixtures with water under the studied reaction conditions. The catalysts were chosen due to their documented lignin depolymerization effect. The oxidants were selected based on their green status. Furthermore, the selection of commonly applied catalysts and oxidants enabled a fair comparison of the current results to the large body of the existing monophasic literature. In addition, $A Q$ was added as a novel acidolytic catalyst so far, only tested with alkaline lignin oxidation. Low loading of the feedstock was chosen to minimize the number of post-conversion sample preparation steps, limiting the sources of experimental errors. 


\section{SI Sample preparation and analysis}

The samples were filtered through a $0.22 \mu \mathrm{m}$ filter and prepared by transferring $180 \mu \mathrm{l}$ of the sample and $20 \mu \mathrm{l}$ of internal standard-vanillin-(phenyl- $\left.{ }^{13} \mathrm{C}_{6}\right)$ into a $0.3 \mathrm{ml}$ chromatography vial and sealed. The chromatographic separation of the analytes was performed with a UHPLC (Dionex Ultimate 3000 Series RS system, Thermo Scientific ${ }^{\mathrm{TM}}$ ), including a pump, a column compartment, and an auto-sampler. HRMS data acquisition was performed using Q-Exactive ${ }^{\mathrm{TM}}$ hybrid quadrupole-orbitrap mass spectrometer (Thermo Scientific $^{\mathrm{TM}}$ ). Mass spectra were acquired in full scan (MS1 and dd-MS2, centroid) mode with an isolation window of $1 \mathrm{~m} / \mathrm{z}$ from $50-750 \mathrm{~m} / \mathrm{z}$ at a maximum resolution of 70'000 for MS1 and 17'500 for dd-MS2. Instruments were calibrated daily. A screening study involving optimization of the LCMS method for optimal instrument performance and analyte response was performed, resulting in the method described in this section. Separation of the analytes was achieved with an RP-MS ${ }^{\mathrm{TM}}$ UHPLC pre-column and column (150 $\mathrm{mm} \times 2.1 \mathrm{~mm}$, particle size $2.1 \mu \mathrm{m}$ ) from Thermo Scientific ${ }^{\mathrm{TM}}$. The temperature of the columns was $50{ }^{\circ} \mathrm{C}$. The following program using mobile phase A $(1 \% \mathrm{ACN}, 1 \%$ $\mathrm{MeOH}$, and $0.2 \% \mathrm{HCOOH}$ in high purity water) and mobile phase $\mathrm{B}(100 \mathrm{vol} . \% \mathrm{MeOH}$ ) at flow rate of $0.700 \mathrm{ml} / \mathrm{min}$ was applied: $1 \% \mathrm{~B}(1 \mathrm{~min})$, from 1 to $99 \% \mathrm{~B}(5 \mathrm{~min})$, and $99 \%$ B ( $2 \mathrm{~min}) .1 \mu \mathrm{l}$ of the prepared solutions were injected. The analytes were ionized in positive mode with ESI (electrospray ionization, spray voltage $3.5 \mathrm{kV}$, and capillary temperature $250{ }^{\circ} \mathrm{C}$ ). Raw mass spectral data files were collected in triplicate for MS1 with an additional MS2 run for identification purposes. Each series of measurements was preceded by the analysis of the baseline samples (converted solvents, assessing the true background of the analyzed chemical space) and followed by a blank assessing possible carry-over during the analysis. The instruments were controlled with $\mathrm{Xcalibur}^{\mathrm{TM}} 4.1$ software. 


\section{SI Raw data processing}

The raw data were processed in Compound Discoverer ${ }^{\mathrm{TM}} 3.0(\mathrm{CD})$, including peak picking (2.5 mass accuracy), retention time alignment ( $0.2 \mathrm{~min})$, and feature componentization within maximum shift alignment of $0.1 \mathrm{~min}$ (grouping of ions, adducts, and isotopes belonging to the same compound). For each peak with a $10^{6}$ counts intensity threshold for precursors in at least one sample, a corresponding peak was targeted in the remaining samples, or a "gap-value" was added in the absence of the peak itself (gap-filling). The composition (of a general formula $\mathrm{C}_{c} \mathrm{H}_{h} \mathrm{O}_{\mathrm{o}} \mathrm{N}_{n} \mathrm{~S}_{\mathrm{s}}$ ) of features was predicted based on exact mass and isotopic patterns. Additionally, the identity of the compounds was determined by a search within mzCloud, and ChemSpider databases, as well as an in-house developed mass list of possible lignin conversion products, developed based on previous work and literature reports. A solvent blank $(\mathrm{MeOH})$ measured with each group of samples was used to mark the background for subtraction in later steps. A process blank of unconverted and converted water, octanol, and water/octanol mixtures was prepared and measured along with the samples. Figure S2 in SI summarizes the raw data processing method. The composition of each measured species was predicted based on the exact mass, fine isotopic patterns, and the available MSMS information. The results were summarized by descriptive statistics and used as an input to differential analysis directly in the Compound Discoverer statistical module as a check of reproducibility and general trends in the data. Further data post-processing was performed to extract more detailed information.

\section{SI Suspect screening and non-targeted analysis}

In case of positive hits during the suspect screening, the validity of each match was assessed manually based on the measured and in-silico MSMS patterns predicted via the Fragment Ion Search algorithm (FISh) in CD. Only The remaining species were evalu- 


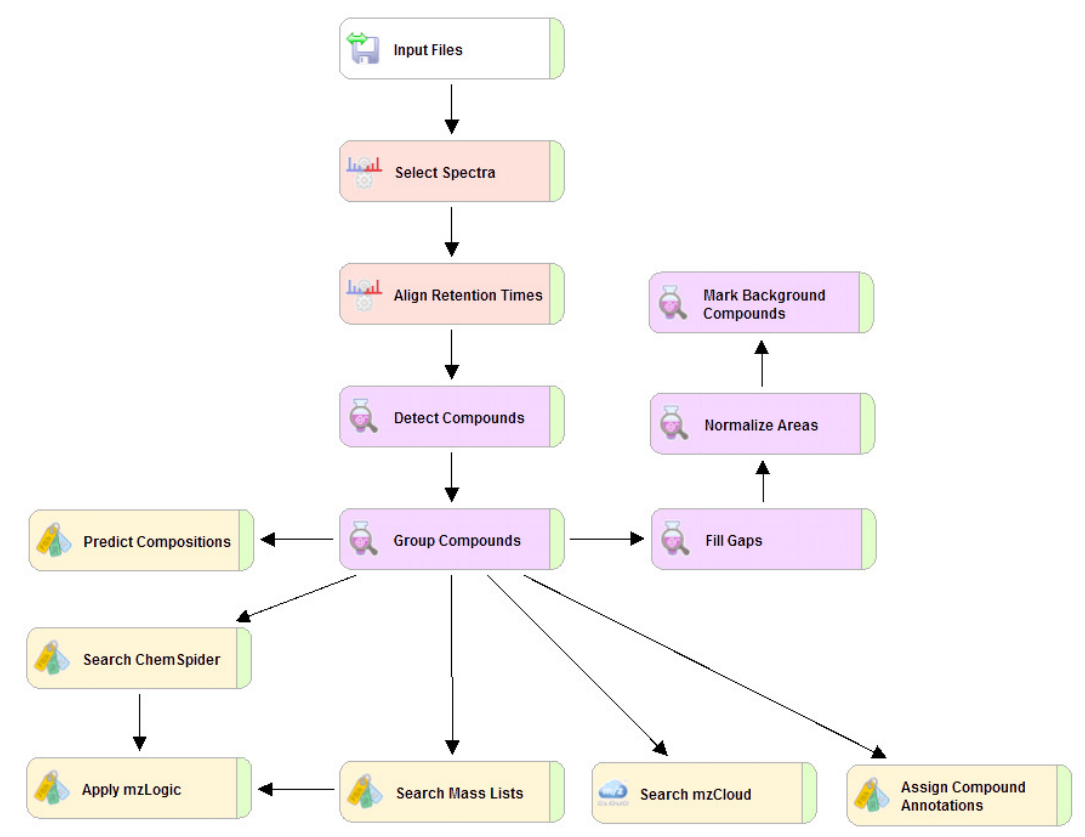

Figure S2: Compound Discoverer untargeted work flow used for deconvolution and processing of the obtained high resolution mass spectrometry data.

ated according to the non-targeted approach. In the first step, the measured MSMS scans were submitted to the mzCloud spectral database for candidate searching. In the case of a lack of a precise spectral match, a similarity search provided the most likely candidates. Subsequently, the masses combined with the predicted compositions, i.e., the chemical formulas, were used as input to several compound mass banks available through ChemSpider. A list of 100 candidates for each species was then evaluated with the mzLogic algorithm scoring each based on its spectral similarity of the experimental MSMS. The likelihood of the top 15 candidates for each compound from mzCloud and/or ChemSpider was assessed with a fragment ion search algorithm (FISh) combining an in-situ candidate fragmentation with automatic scoring of the resulting fragmentation patterns against the experimental values for a given feature. Only species with either a high mzCloud or FISh score, preferably both, were assigned high-probability level molecular identifications. 


\section{SI Data export and processing}

Result data files were exported from CD as csv files, imported, cleaned up, processed, and analyzed in KNIME Analytics Platform (4.3.2.v202103051237), including native KNIME nodes, open-source nodes for R and Python scripting, licensed ChemAxon JChem nodes for molecule visualization, as well as cheminformatics open source nodes such as CDK, RDkit, Vernalis, Indigo, and Erlwood. The workflows can be made available upon request.

\section{SI Statistics and data mining}

The trends in obtained yields were assessed by appropriate univariate analyses (OneWay ANOVA, Two-Way ANOVA, Pearson correlation), and various machine learning and data mining methods, including unsupervised clustering (hierarchical and k-means), data reduction via principal component analysis (PCA), and supervised tree ensemble methodology. Yields of single identified monomers, yields of all monomers summed per run, as well as yields per class per run, were applied as input to the analyses, except for the PCA analysis, which has shown a much-improved performance with areas as input. Section lists the respective compound lists with properties, yields, and IDs. ANOVA and correlation analyses, which were used to evaluate the univariate trends in the yields as a function of phase (water and octanol), oxidant, and catalyst, were assessed with pvalues and visualized via box plots and heat-maps. Hierarchical (Ward method, explored via a dendrogram) and k-means (clusters selected based on Elbow analysis method, $\mathrm{k}$ =3) clustering were used to group similar samples based on multivariate trends in the yields. Principal component analysis (PCA) was used to reduce the dimensionality of the data and observe the emerging hidden trends. PCA is a multivariate data analysis procedure that uses an orthogonal transformation of possibly correlated variables into a set of linearly uncorrelated principal components (PCs). PC1 represents the direction 
of most significant variance in the data, PC2 the second largest, and so on with the only constraint that each PC is orthogonal to the other components. The results were evaluated by score plots (projection of the samples to the principal components) and loading plots (contribution of each variable to the orientation of the PCs). A Tree Ensemble Learner (number of models 100, random sampling with replacement, a different set of attributes is used for each model, regression) from KNIME was used to train tree-based predictive models for all identified aromatic monomers. The models were evaluated based on $\mathrm{R}^{2}$ values as well as the measured vs. predicted plots.

\section{SI Results}

Overall yields of classes Due to the differences in ionization efficiencies (IE) between the applied standard (vanillin) and aliphatics, cyclics, lignin, and condensates, the calculations of those species yields are purely qualitative for the sake of comparison.
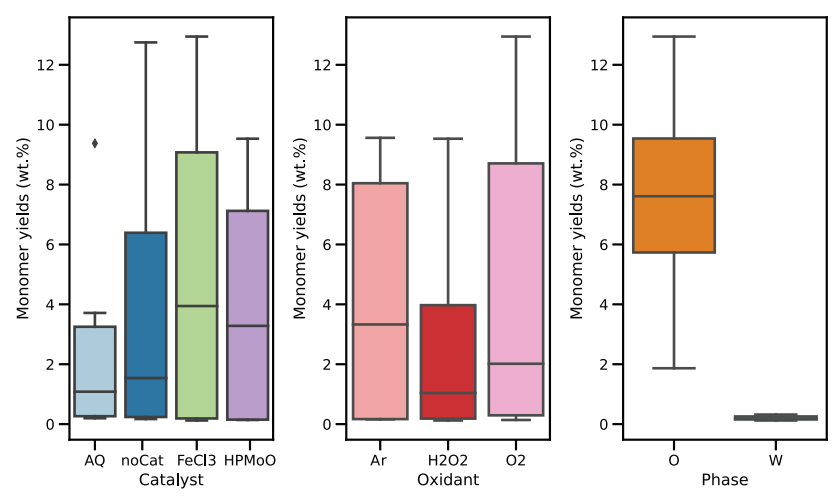

Figure S3: Box plot showing the overall monomeric yields in all the samples as the function of phase (water, octanol), oxidant, and catalyst. 

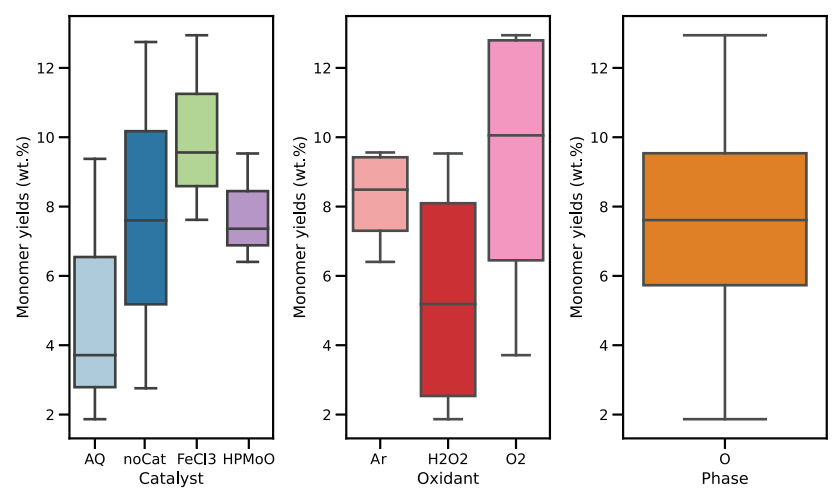

Figure S4: Box plot showing the overall monomeric yields in the octanol samples as a function of oxidant and catalyst. While the line inside the box indicates the measured median, its boundaries represent Q1 to Q3 quartiles (50\% of the values). The whiskers show the max and the min yields.
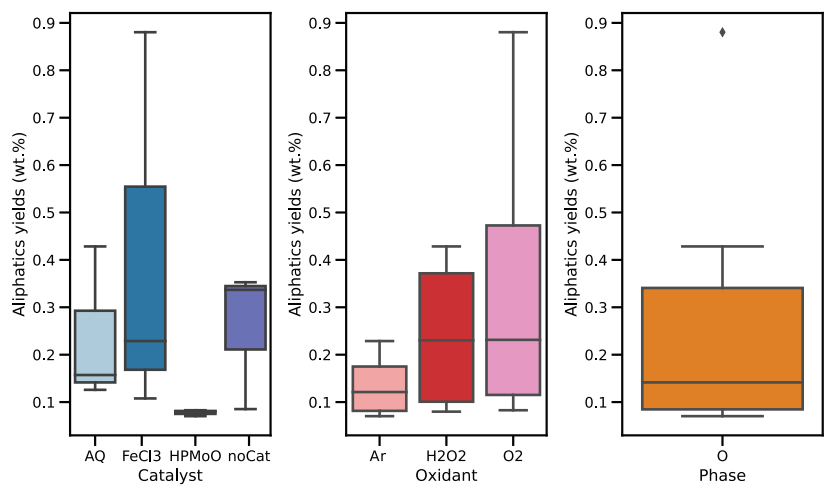

Figure S5: Box plot showing the overall aliphatic yields in the octanol samples as the function of oxidant and catalyst. While the line inside the box indicates the measured median, its boundaries represent Q1 to Q3 quartiles (50\% of the values). The whiskers show the max and the min yields. Diamond-shaped points indicate outliers.
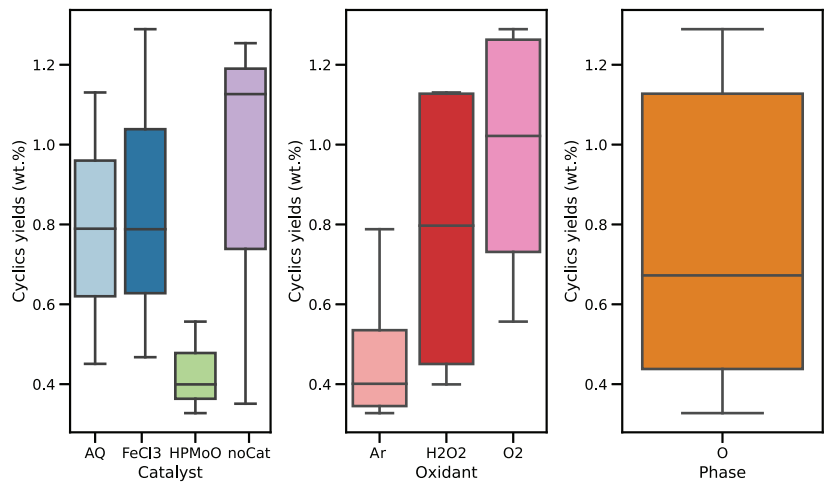

Figure S6: Box plot showing the overall cyclics yields in the octanol samples as the function of oxidant and catalyst. 

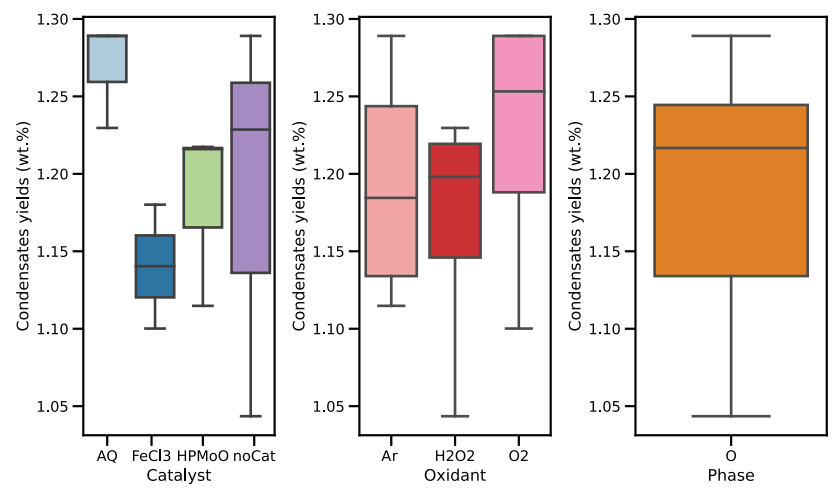

Figure S7: Box plot showing the overall condensate yields in the octanol samples as the function of oxidant and catalyst.
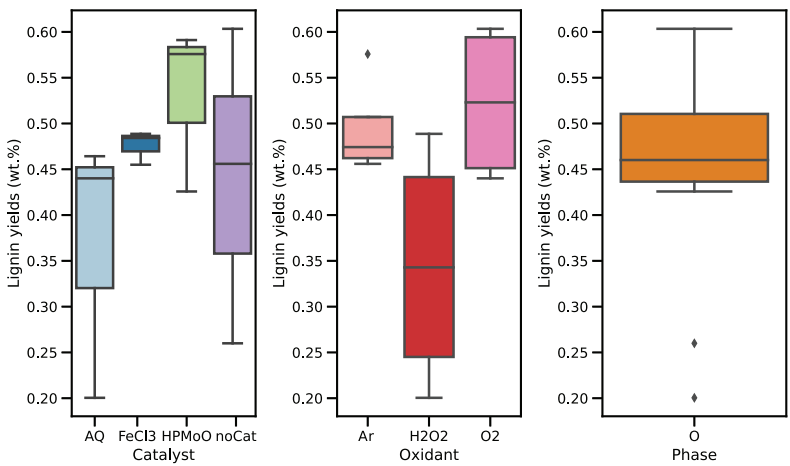

Figure S8: Box plot showing the overall lignin fragments yields in the octanol samples as the function of oxidant and catalyst.

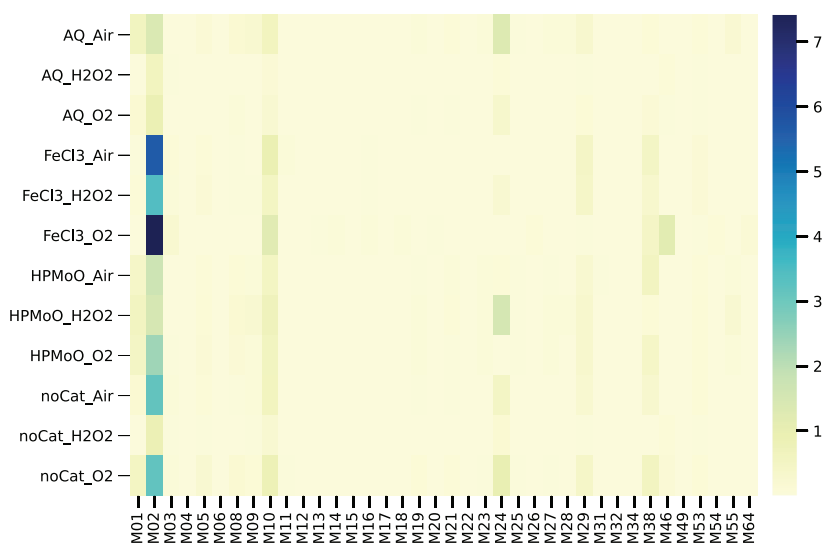

Figure S9: Matrix plot comparing the yields of the identified monomers (M-ID were summarized in SI file filename) in octanol samples across all runs. 


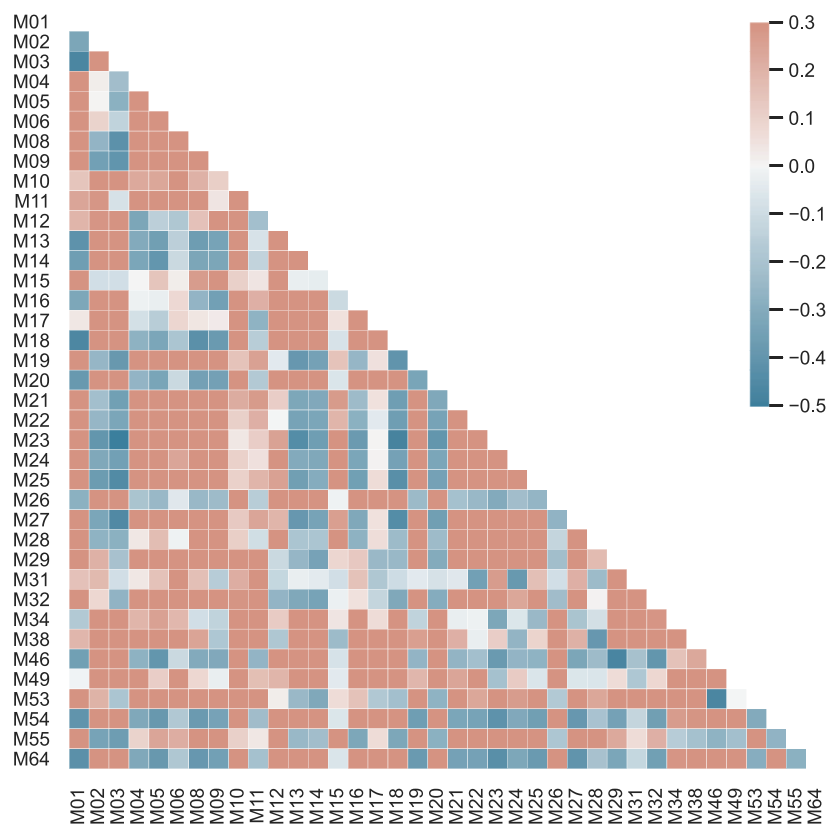

Figure S10: Correlations between the yields of the identified monomers visualized as a heat-map. Minor to moderate correlation coefficients were observed.

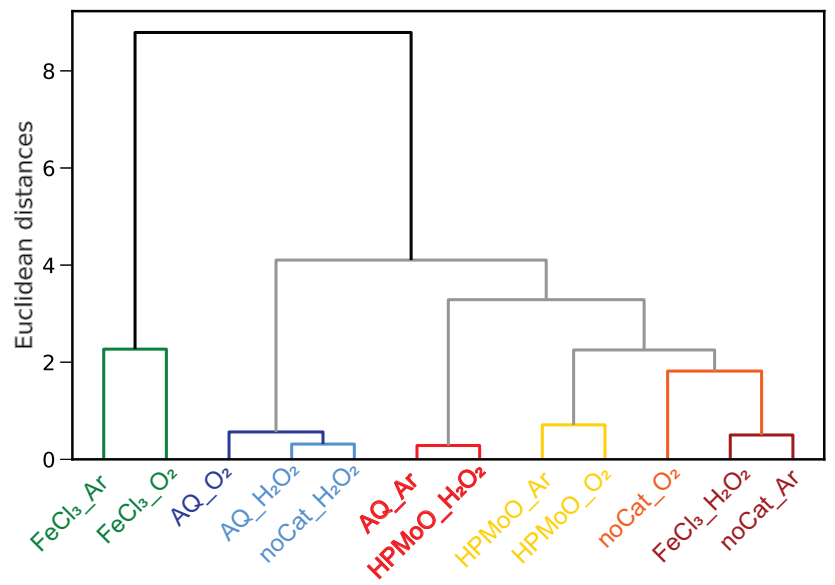

Figure S11: Results from the hierarchical clustering of the octanol samples based on their aromatic monomer yields. Computed with scipy hierarchy module in KNIME. 


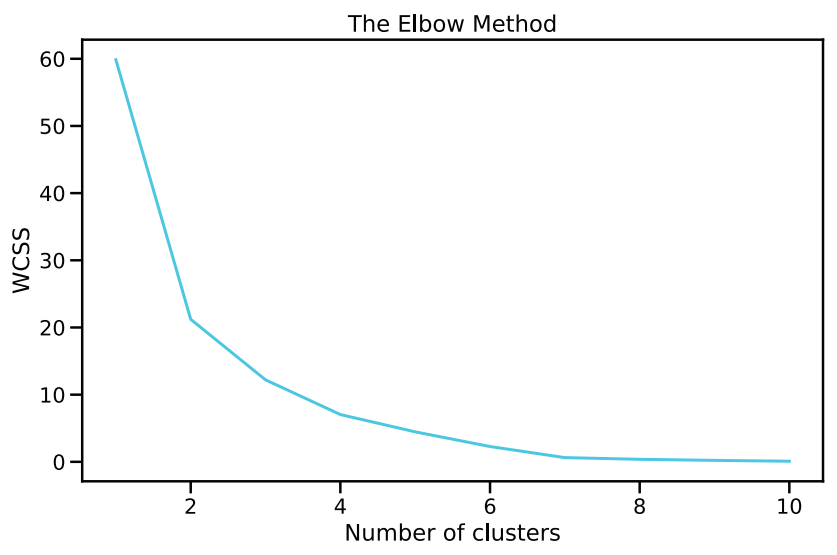

Figure S12: Elbow plot used for finding the optimal number of clusters (between 1 and 10) for $k$-means clustering, in this case between $k=3$ and $k=4$. Based on minimization of WCSS (within cluster sum of squares) between aromatic monomer yields from different runs. Computed with sklearn KMeans module in KNIME. 


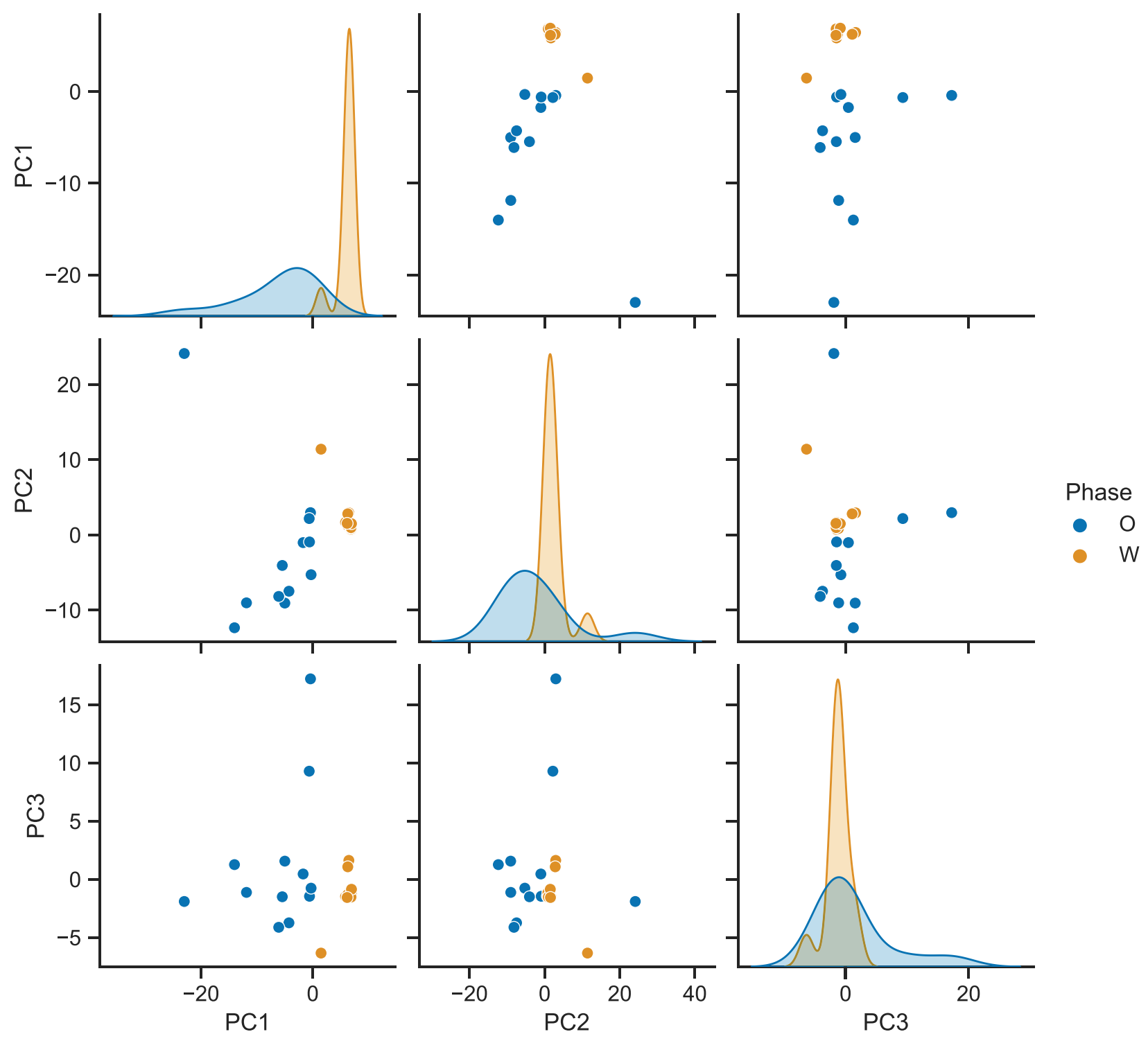

Figure S13: PCA score plots for all samples colored according to the phase. 

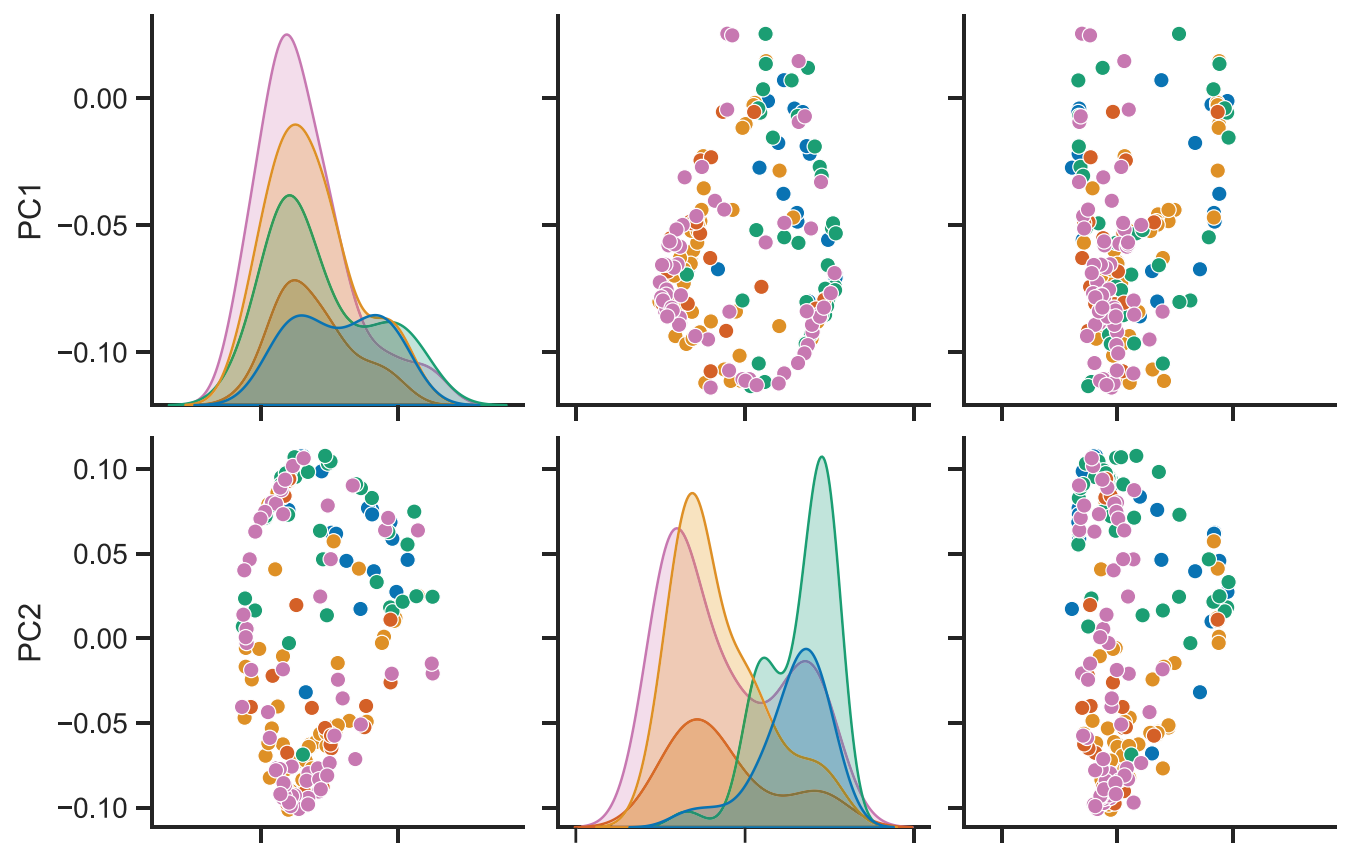

Class

- Aliphatic

- Condensate

- Cyclic

- Lignin

- Monomer
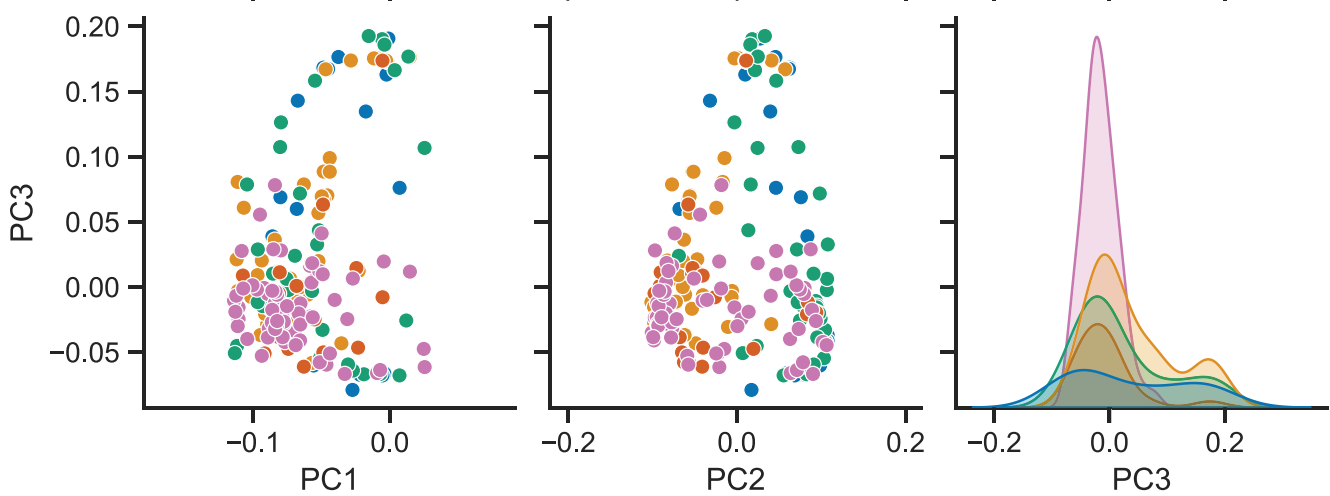

Figure S14: PCA loading plots for all samples colored according to the class. 

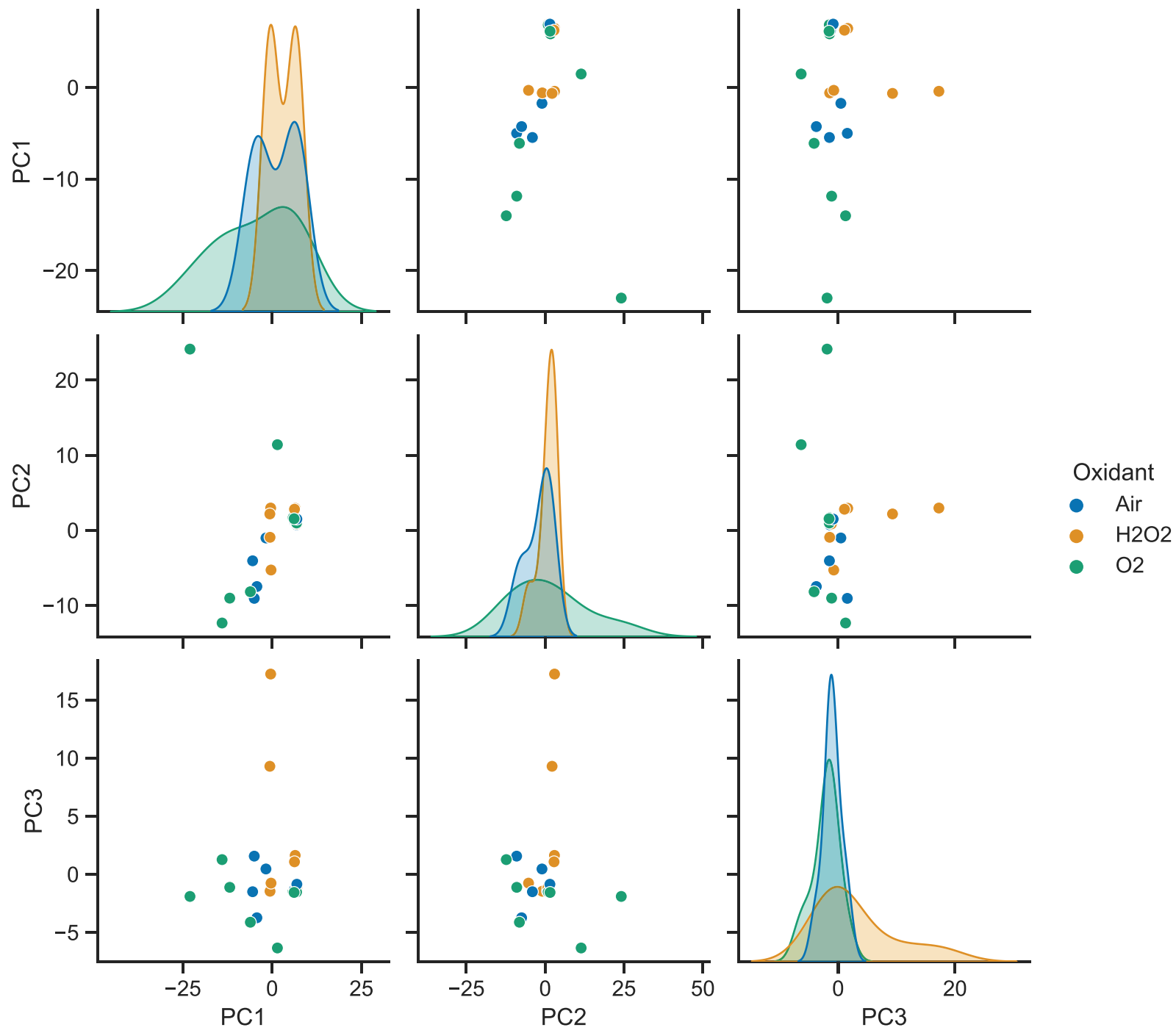

Figure S15: PCA score plots for all samples colored according to the oxidant. PC1 (31\%), PC2 (26 \%), PC3 (10 \%). 7 PCs necessary to explain $90 \%$ of the variability in the data. 

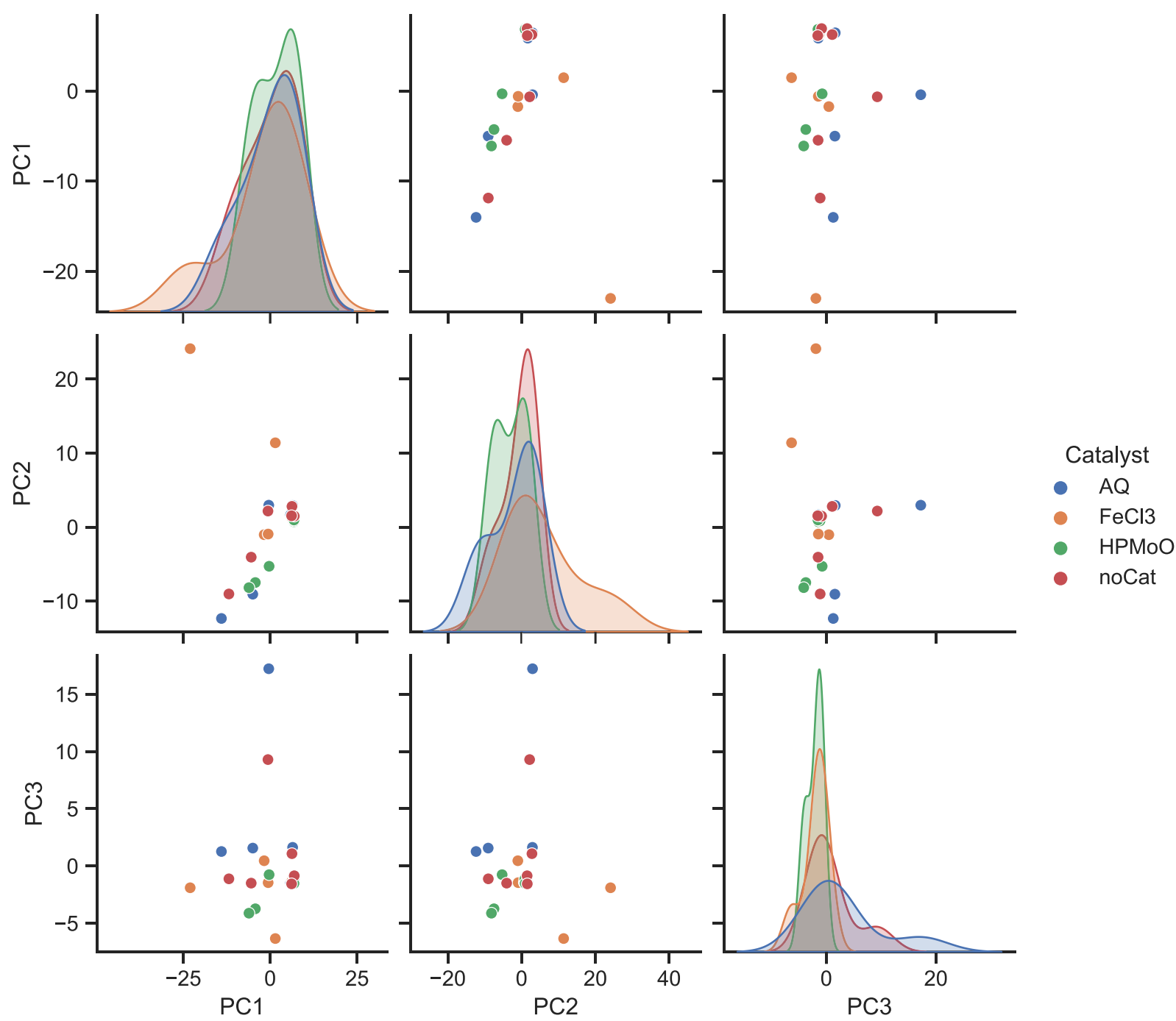

Figure S16: PCA score plots for all samples colored according to the catalyst. 


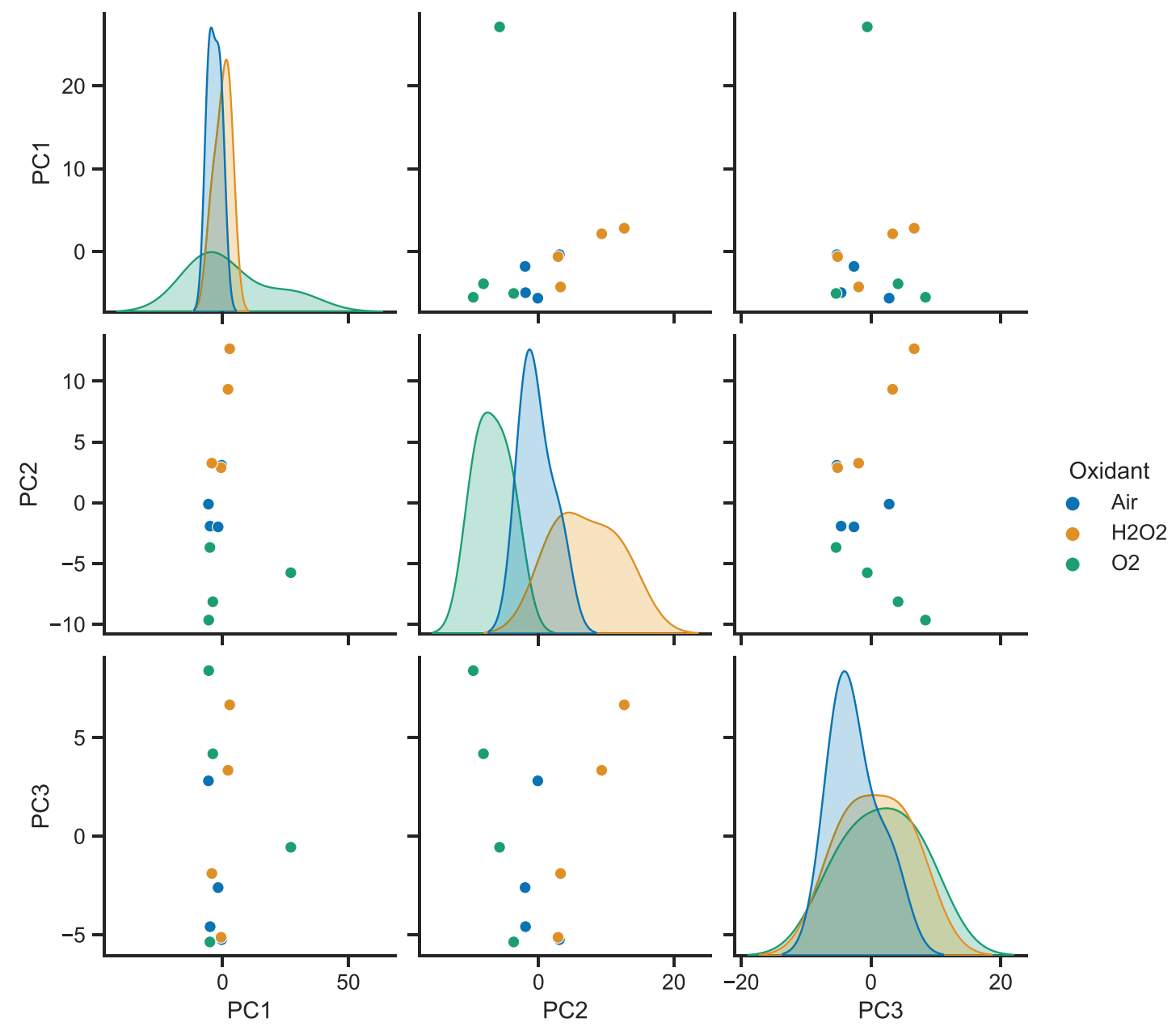

Figure S17: PCA score plots for octanol samples colored according to the oxidant. PC1 (40 \%), PC2 (22 \%, PC3 (12\%). 5 PCs necessary to explain $90 \%$ of the variability in the data. 

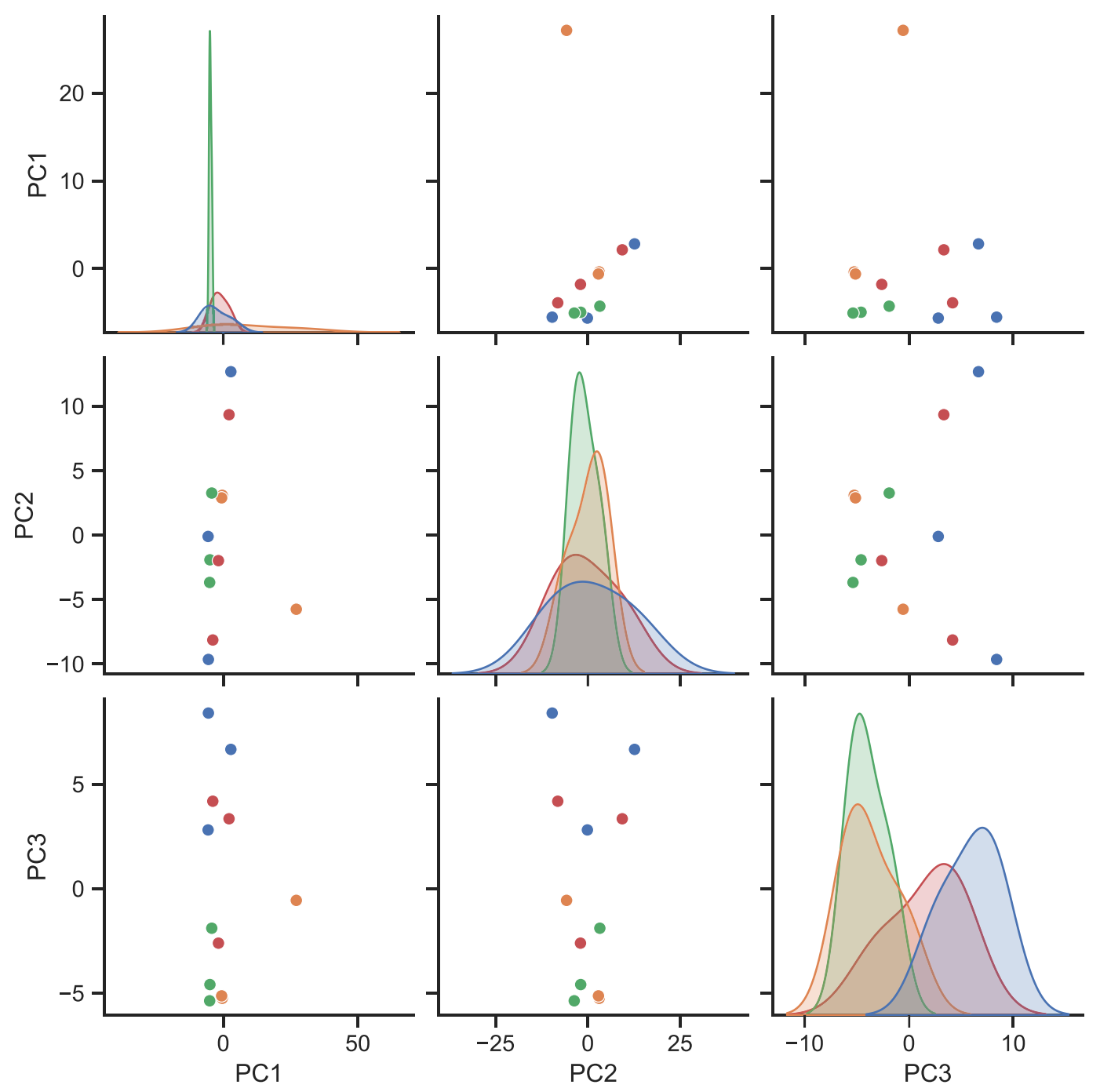

Catalyst

- $A Q$

- $\mathrm{FeCl} 3$

- HPMoO

- noCat
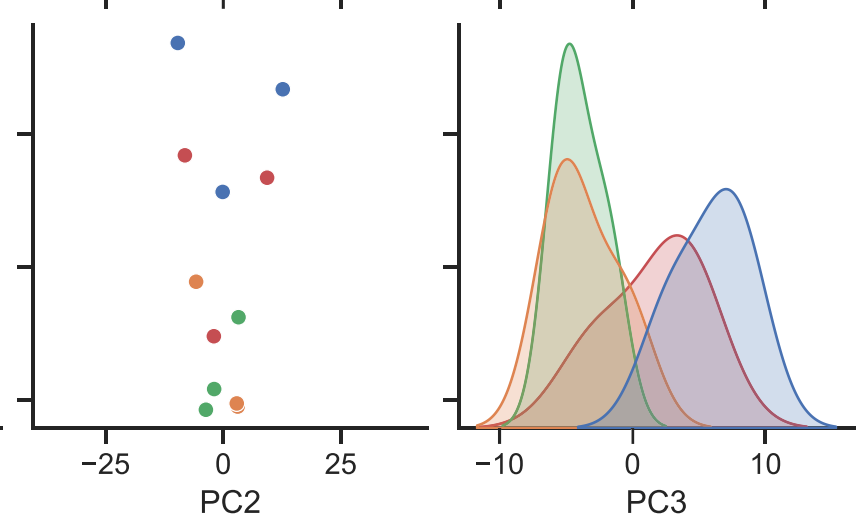

Figure S18: PCA score plots for octanol samples colored according to the catalyst. PC1 $(40 \%)$, PC2 (22 \%), PC3 (12\%). 5 PCs necessary to explain $90 \%$ of the variability in the data. 

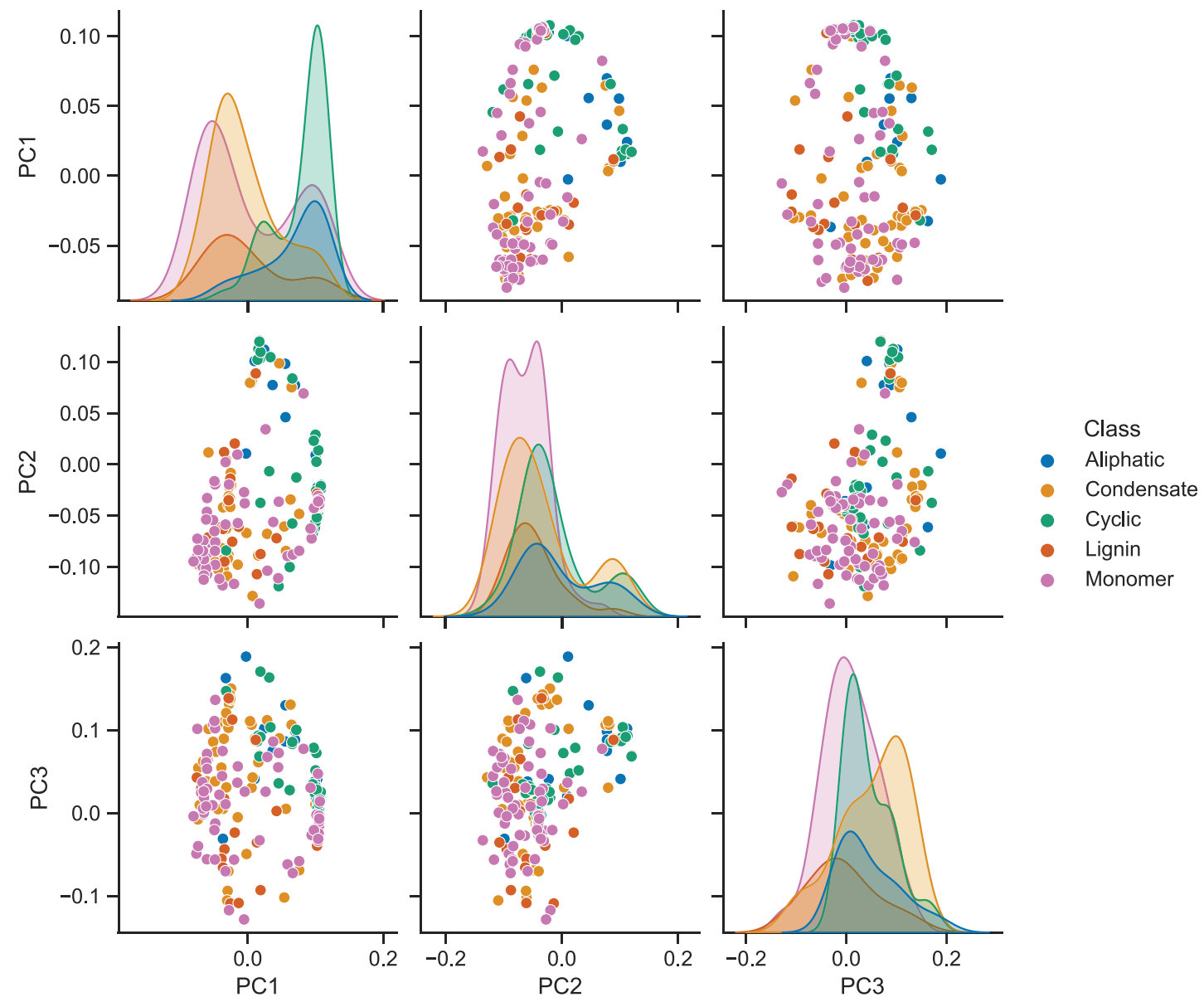

Figure S19: PCA loading plots for octanol samples colored according to the class. 

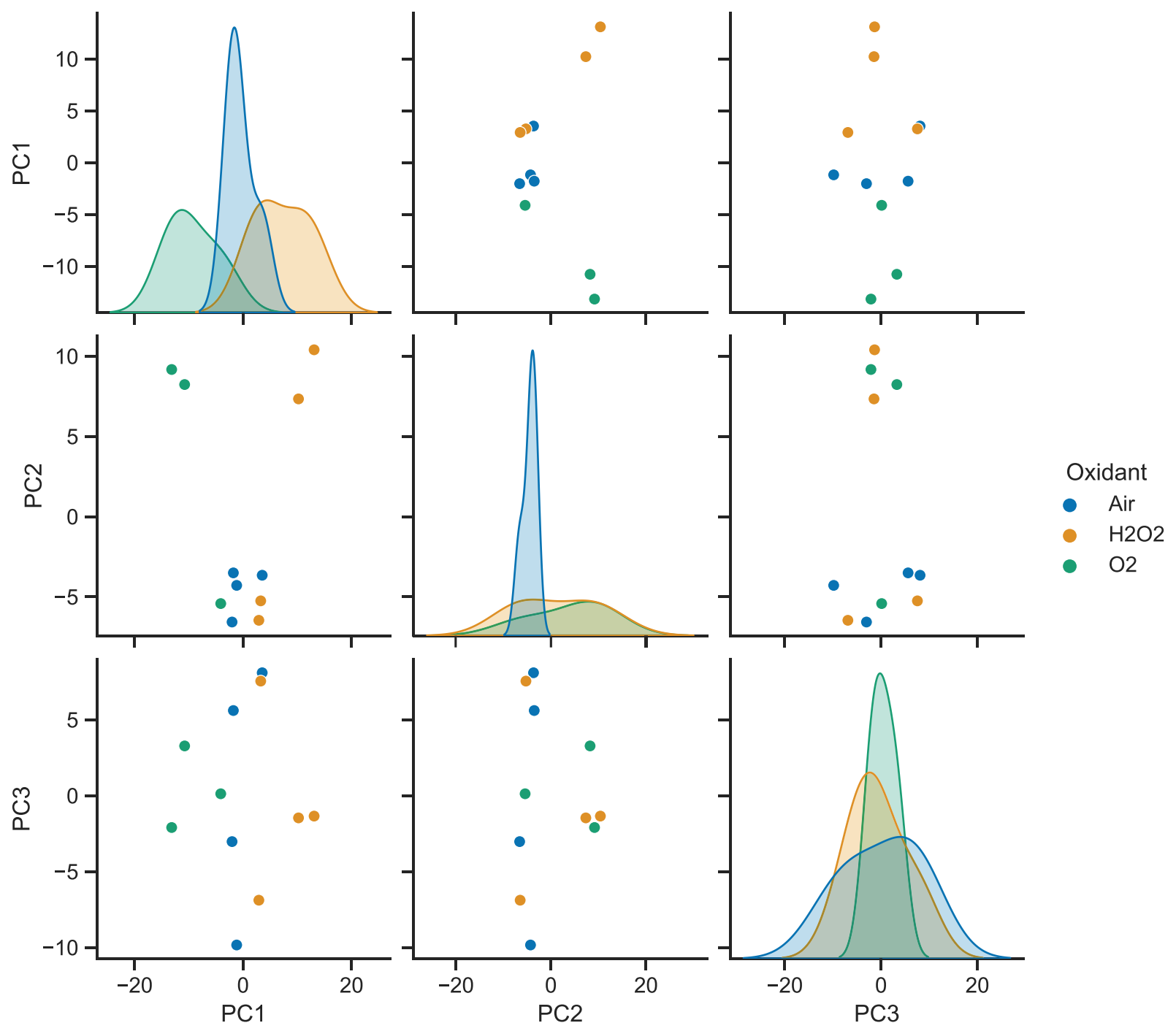

Figure S20: PCA score plots for octanol samples (except run $\mathrm{R}_{\mathrm{k}}$ ) colored according to the oxidant. PC1 (30\%), PC2 (25\%), PC3 (16\%). 6 PCs necessary to explain $90 \%$ of the variability in the data. 


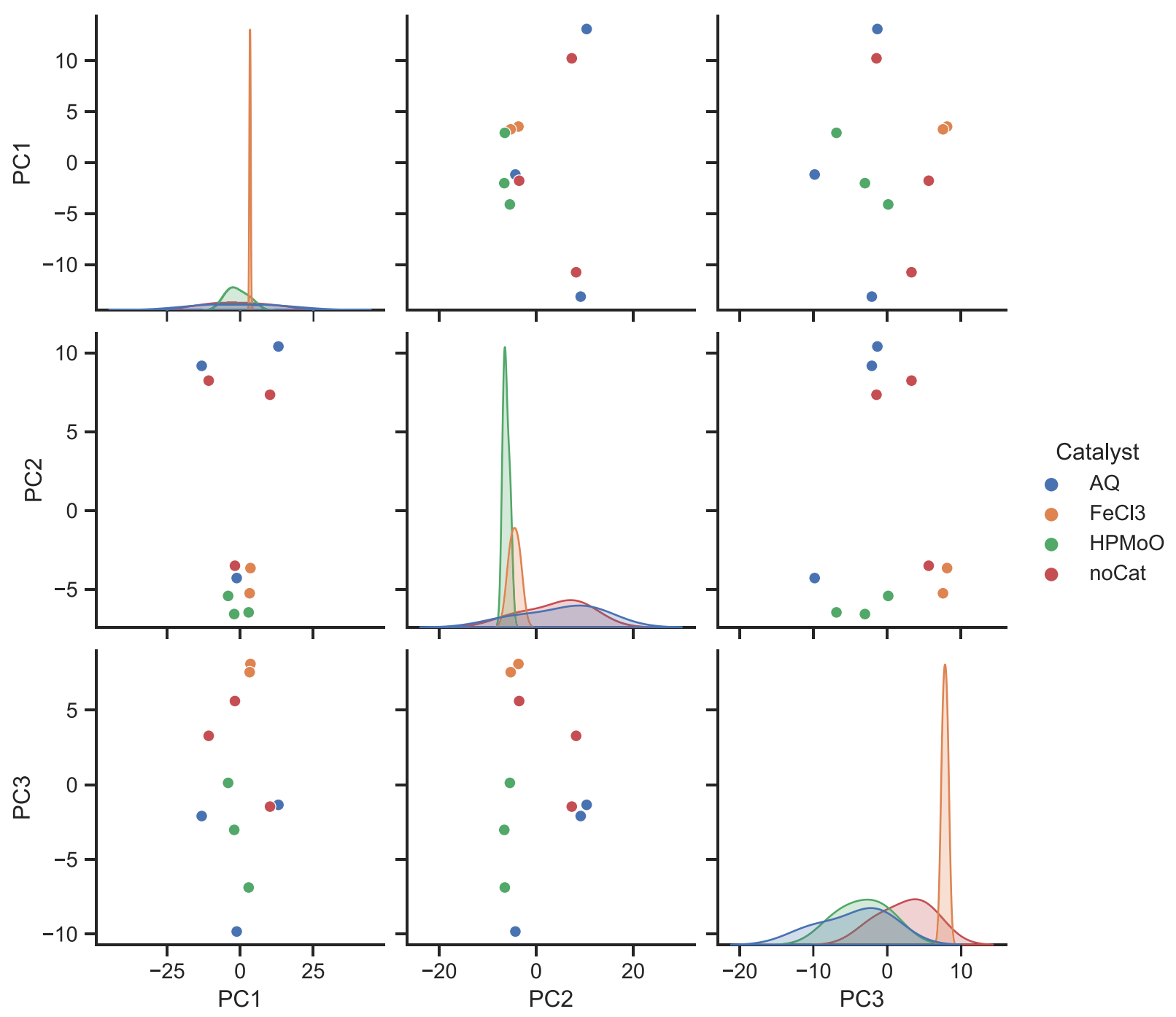

Figure S21: PCA score plots for octanol samples (except run $\mathrm{R}_{\mathrm{k}}$ ) colored according to the catalyst. 

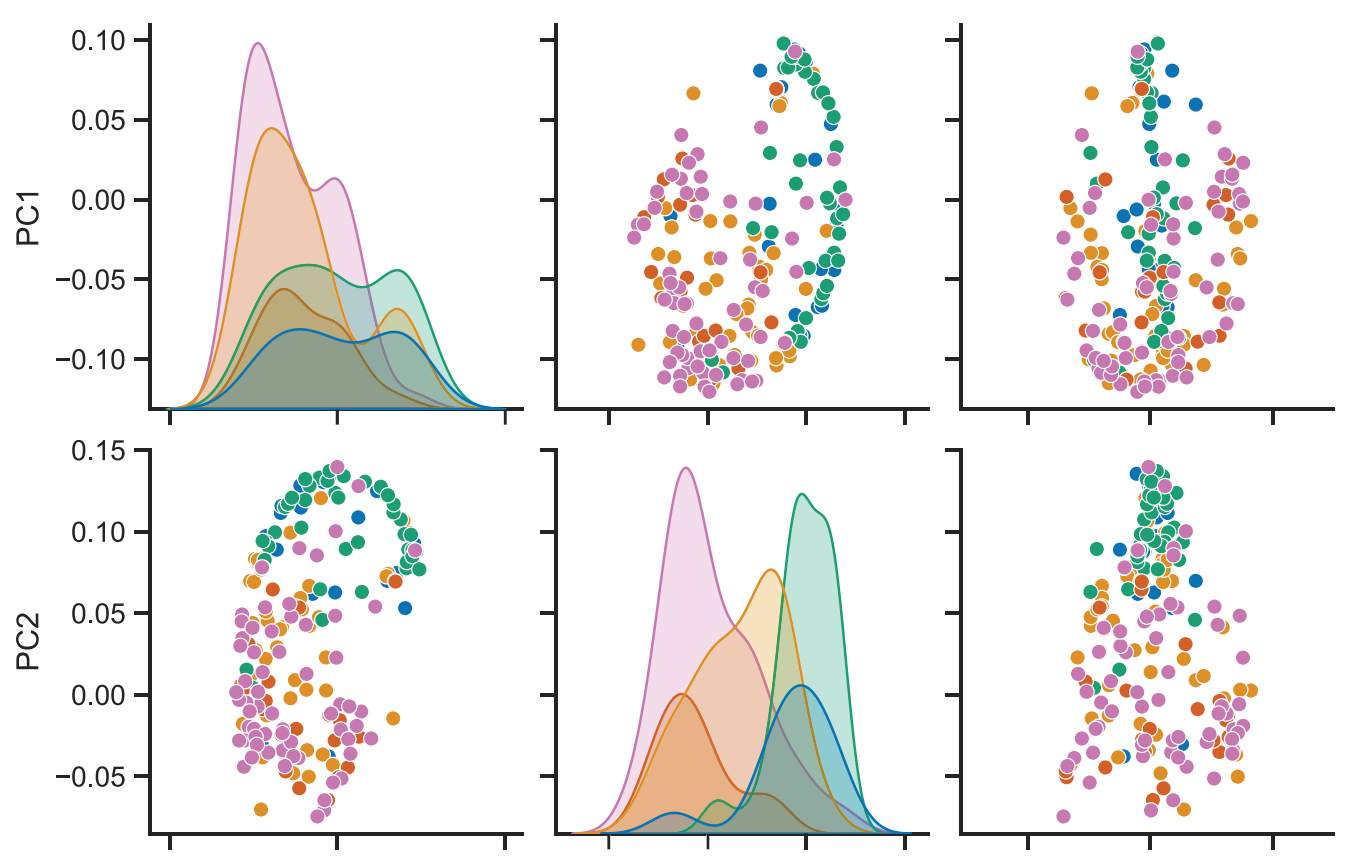

Class

- Aliphatic

- Condensate

- Cyclic

- Lignin

- Monomer
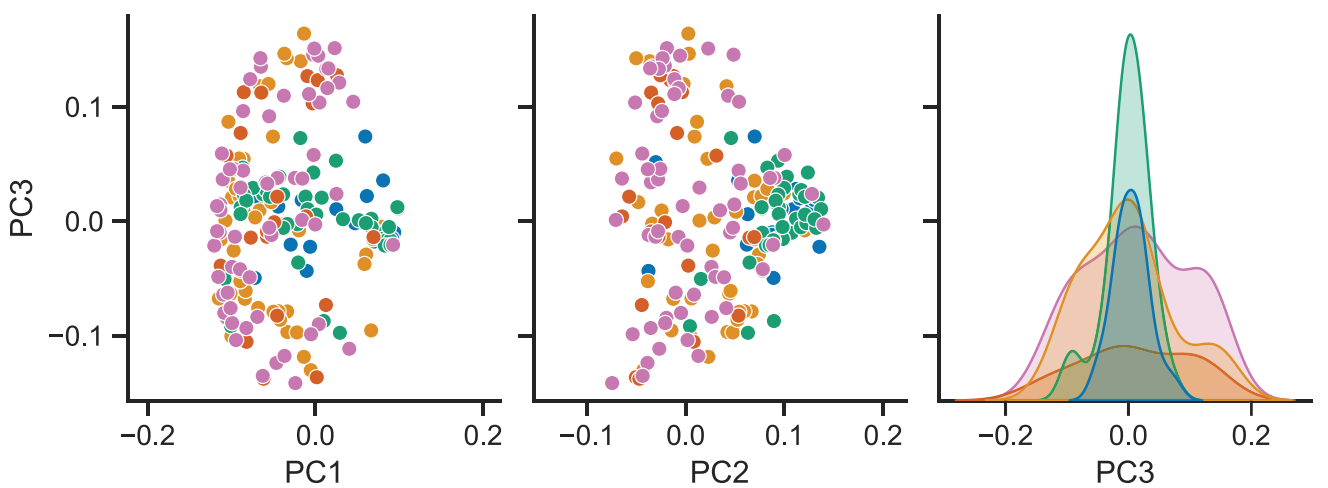

Figure S22: PCA loading plots for octanol samples (except run $\mathrm{R}_{\mathrm{k}}$ ) colored according to the class. 